\title{
On Hawking Radiation as Tunneling with Logarithmic Corrections
}

\author{
A.J.M. Medved ${ }^{a}$ and Elias C. Vagenas ${ }^{b}$ \\ ${ }^{a}$ School of Mathematics, Statistics and Computer Science \\ Victoria University of Wellington \\ PO Box 600, Wellington, New Zealand \\ E-Mail: joey.medved@mcs.vuw.ac.nz \\ ${ }^{b}$ Nuclear and Particle Physics Section \\ Physics Department \\ University of Athens \\ GR-15771, Athens, Greece \\ E-Mail: evagenas@phys.uoa.gr
}

November 18, 2018

\begin{abstract}
There has been recent speculation that the tunneling paradigm for Hawking radiation could - after quantum-gravitational effects have suitably been incorporated - provide a means for resolving the (black hole) information loss paradox. A prospective quantum-gravitational effect is the logarithmic-order correction to the Bekenstein-Hawking entropy/area law. In this letter, it is demonstrated that, even with the inclusion of the logarithmic correction (or, indeed, the quantum correction up to any perturbative order), the tunneling formalism is still unable to resolve the stated paradox. Moreover, we go on to show that the tunneling framework effectively constrains the coefficient of this logarithmic term to be non-negative. Significantly, the latter observation implies the necessity for including the canonical corrections in the quantum formulation of the black hole entropy.
\end{abstract}


The intuitive notion of Hawking radiation [1] as a quantum tunneling event (e.g., [2-4]) - along with the fundamental principle of energy conservation - provides a meticulous framework for incorporating back-reaction effects into the black hole radiative process. To elaborate, the positive-energy particle of a spontaneously produced pair (formed just inside of the black hole horizon) can quantum tunnel its way out of the horizon to infinity; with energy conservation then being enforced by the negative-energy partner. ${ }^{1}$ Even at the tree level (i.e., sans back-reaction effects), this tunneling paradigm can correctly reproduce the Hawking temperature for a myriad of black hole and de Sitter spacetimes; see, for instance, [5-7] and [8] for many additional references. Moreover, the inclusion of the back-reaction leads to the distinct quantitative prediction of a non-thermal emission spectrum. Such a spectrum is an intriguing result; being suggestive of a "loophole" in the usual argument for the loss of black hole information. That is, the so-called (black hole) information loss paradox [9] is, as typical of many relevant discussions [10], predicated on the idea of a purely thermal spectrum.

It is normal to associate lost information with a non-unitary quantum theory. Hence, it is of further significance (with regard to the stated paradox) that the tunneling paradigm directly implies a unitary process of black hole evaporation [11,12]. Let us, on this point, be more explicit and consider one of the key outcomes of the tunneling methodology: Namely, the tunneling probability or emission rate can consistently be cast into the suggestive form (e.g., $[5,13])$

$$
\Gamma \sim \exp [\Delta S] .
$$

(Here, $S$ denotes the Bekenstein-Hawking black hole entropy $[1,14]$ or, more generally, the horizon entropy in the case of de Sitter spacetimes [15].) That is, the logarithm of this probability is (modulo an irrelevant constant) equal to the change in the black hole entropy during the radiative process. Just such an outcome complies perfectly with statistical considerations (i.e., $\ln \Gamma \sim$ final density of states/initial density of states) and thus implies a unitarily evolving system.

In spite of the non-thermal spectrum and implied unitarity, it is not at all clear (as first pointed out by Parikh [11]) as to how the information might actually be preserved. The obvious answer - namely, in correlations between emitted particles - is problematic in that such correlations do not appear to exist. ${ }^{2}$ One can see that this is the case by observing the following (see [11] and below for details):

$$
\ln \left[\Gamma\left(E_{1}\right) \Gamma\left(E_{2}\right)\right]=\ln \left[\Gamma\left(E_{1}+E_{2}\right)\right],
$$

where each probability has been expressed as a function of the emitted particle energy. To put it another way, the probability of emitting two particles of energy $E_{1}$ and $E_{2}$ is exactly the same as the probability of emitting just a single particle of the same total energy; meaning that these probabilities (and the emitted particles themselves) are then necessarily uncorrelated. And so, given that the desired correlations are non-existent to begin with, it is impractical to suggest that these could serve as a conduit for the information.

\footnotetext{
${ }^{1}$ Note that the same basic idea applies to a de Sitter cosmological horizon, except that (in this case) the positive-energy particle will tunnel inwards to an appropriate de Sitter observer.

${ }^{2}$ At least at late times. The tunneling methodology, as it currently stands, can not yet address the issue of short-time correlations.
} 
On the other hand, a possible resolution to this paradoxical situation has recently been proposed by Arzano [16]. This author has suggested that, instead of using the conventional (tree-level) Bekenstein-Hawking entropy or ${ }^{3}$

$$
S=\frac{A}{4}
$$

one should rather utilize its quantum-corrected formulation. More specifically,

$$
S_{q}=\frac{A}{4}+\alpha \ln \left[\frac{A}{4}\right]+\text { const. }+\mathcal{O}\left[A^{-1}\right]
$$

where $\alpha$ is a model-dependent (dimensionless) parameter that reflects our ignorance of the fundamental theory of quantum gravity. That the quantum-corrected entropy does take on just such a form has been demonstrated frequently in the literature; see, for example, [17-19] and [20] for many other pertinent citations. Most notably, loop quantum gravity predicts (for a Schwarzschild black hole) a microcanonical contribution to $\alpha$ of $-1 / 2[21,22]$. Given that the black hole has somehow equilibrated with a suitable heat bath, there would also be an additional canonical contribution of at least $+1 / 2$ (e.g., [23-25]). For a recent discussion on this distinction between microcanonical and canonical contributions to the logarithmic prefactor, see [26].

To better understand the underlying premise - and then, alas, the failure - of the proposed resolution, let us focus on the simple case of a Schwarzschild black hole (even though the formal machinery is much more general). Firstly, if a black hole of initial mass $M$ emits a particle of energy $E_{1}$, it follows that the associated probability is given by $[c f$, $A(M)=16 \pi M^{2}$ and equation (10]

$$
\ln \left[\Gamma\left(M ; E_{1}\right)\right]=S\left(M-E_{1}\right)-S(M)=-8 \pi M E_{1}\left(1-\frac{E_{1}}{2 M}\right) ;
$$

where we have, for the moment, chosen to ignore the possibility of quantum corrections. A second emission of a particle of energy $E_{2}$ will then occur with a probability of

$$
\ln \left[\Gamma\left(M-E_{1} ; E_{2}\right)\right]=S\left(M-E_{1}-E_{2}\right)-S\left(M-E_{1}\right)=-8 \pi\left(M-E_{1}\right) E_{2}\left(1-\frac{E_{2}}{2\left(M-E_{1}\right)}\right) .
$$

The reader should have no trouble verifying that the sum of equations (5) and (6) coincides exactly with the calculation of $\Gamma\left(M ; E_{1}+E_{2}\right)=S\left(M-E_{1}-E_{2}\right)-S(M)$; which is really just a restatement of equation (21).

Now let us, as initially suggested by Arzano [16], repeat the computation for the quantum-corrected entropy $S_{q}$ (up to the logarithmic order). The first emission gives us

$$
\begin{aligned}
\ln \left[\Gamma_{q}\left(M ; E_{1}\right)\right] & =S_{q}\left(M-E_{1}\right)-S_{q}(M) \\
& =-8 \pi M E_{1}\left(1-\frac{E_{1}}{2 M}\right)+2 \alpha \ln \left[\frac{M-E_{1}}{M}\right] .
\end{aligned}
$$

Then, the second emission yields the result

$$
\begin{aligned}
\ln \left[\Gamma_{q}\left(M-E_{1} ; E_{2}\right)\right] & =S_{q}\left(M-E_{1}-E_{2}\right)-S_{q}\left(M-E_{1}\right) \\
& =-8 \pi\left(M-E_{1}\right) E_{2}\left(1-\frac{E_{2}}{2\left(M-E_{1}\right)}\right)+2 \alpha \ln \left[\frac{M-E_{1}-E_{2}}{M-E_{1}}\right] .
\end{aligned}
$$

\footnotetext{
${ }^{3}$ A note on conventions: We will be setting all fundamental constants equal to unity and $A$ denotes
} the cross-sectional area of the black hole (or cosmological) horizon under scrutiny. 
Summing these two revised outcomes, we obtain a (combined) logarithmic term of simply $2 \alpha \ln \left[\frac{M-E_{1}-E_{2}}{M}\right]$. It should be reasonably clear that precisely the same logarithmic term arises out of $\Gamma_{q}\left(M ; E_{1}+E_{2}\right)$; that is, the probability for a single-particle emission of the same total energy. Consequently, the quantum analogue of equation (2), or

$$
\ln \left[\Gamma_{q}\left(E_{1}\right) \Gamma_{q}\left(E_{2}\right)\right]=\ln \left[\Gamma_{q}\left(E_{1}+E_{2}\right)\right],
$$

must certainly be true (at least) to logarithmic order. In fact, after just a few more iterations, it is not difficult to convince oneself that this result must be true up to any order of the power-law expansion implied by equation (4). Hence, the inclusion of such corrections is not sufficient to account for the late-time correlations. Which is to say, even with the quantum (gravitationally) corrected entropy, the tunneling formalism can still not provide a mechanism for preserving the black hole information.

Also of interest, Arzano made a pertinent observation which essentially goes as follows [16]: The logarithmic-correction term implies that $\Gamma \rightarrow 0$ as $E \rightarrow M$ and, as a consequence, provides a natural way of suppressing emission as the energy of the emitted particle approaches the initial mass of the black hole. Such a suppression can indeed occur; however, as has previously gone unnoticed, this can only be the case if the parameter $\alpha$ is positive. This constraint becomes quite evident when equation (17) is exponentiated (now dropping the subscript on the particle energy):

$$
\Gamma_{q}(M ; E)=\left(1-\frac{E}{M}\right)^{2 \alpha} \exp \left[-8 \pi M E\left(1-\frac{E}{2 M}\right)\right] .
$$

Clearly, the logarithmic correction will suppress "black hole sized" emissions (i.e., $E \rightarrow$ $M$ ) when $\alpha>0$ but will have just the opposite effect when $\alpha<0$. That is, a negative value of $\alpha$ will cause the emission probability to diverge in this limit!

Actually, this outcome is not much of a surprise, given that an evaporating Schwarzschild black hole is (when in isolation) a highly unstable system; with this instability being a direct consequence of a negative heat capacity. To achieve stability, it is necessary to immerse the black hole in a suitable heat bath; allowing the system to equilibrate until the temperature of the bath attains the same value as that of the Hawking radiation. (To realize this type of scenario, one could either place the black hole in a reflective "box" or, more pragmatically, create the effects of such a box by working in an anti-de Sitter spacetime.) Notably, the correct description of such a system is provided by the canonical ensemble. Hence, it should also not come as much of a surprise (and is quite reassuring) that canonical corrections appear to be sufficient to ensure a strictly non-negative value for $\alpha[26]$.

To summarize, we have shown (in the context of the tunneling model for Hawking radiation) that the logarithmic correction to the black hole entropy is unable to neither resolve the information-storage problem nor suppress black hole sized emissions in a purely microcanonical framework. The best way to understand these outcomes is, in our opinion, as follows: Although the tunneling paradigm and the quantum-corrected entropy can be viewed as manifestations of quantum-gravitational principles, both of these have (in their current guise) been formulated at only the level of semi-classical gravity. ${ }^{4} \mathrm{~A}$ truly

\footnotetext{
${ }^{4}$ It is probably relevant that, as Page has convincingly argued, the storage of information in the correlations could neither be confirmed nor excluded by a perturbative analysis [27]. Presumably, any semi-classical calculation would fall into this ambiguous class.
} 
quantum-gravitational treatment would, in all likelihood, resolve these (and many other) issues; but it is probably fair to say that no such treatment is promptly forthcoming. Nevertheless, even semi-classical gravity provides much "food for thought", and we commend Arzano (in spite of our critical observations) for initiating a promising direction of investigation.

\section{Addendum}

It should be noted that M. Arzano has since acknowledged our findings and will be adjusting the paper [16] accordingly. Furthermore, we anticipate a collaboration with M. Arzano that encompasses both the current work and [16].

\section{Acknowledgments}

Research for AJMM is supported by the Marsden Fund (c/o the New Zealand Royal Society) and by the University Research Fund (c/o Victoria University). The work of ECV is financially supported by the PYTHAGORAS II Project "Symmetries in Quantum and Classical Gravity" of the Hellenic Ministry of National Education and Religions.

\section{References}

[1] S.W. Hawking, Nature 248, 30 (1974);

Commun. Math. Phys. 43, 199 (1975).

[2] P. Kraus and F. Wilczek, Nucl. Phys. B 433, 403 (1995) gr-qc/9408003;

Nucl. Phys. B 437, 231 (1995) hep-th/9411219.

[3] E. Keski-Vakkuri and P. Kraus, Phys. Rev. D 54, 7407 (1996) hep-th/9604151.

[4] K. Srinivasan and T. Padmanabhan, Phys. Rev. D 60, 024007 gr-qc/9812028;

S. Shankaranarayanan, K. Srinivasan, and T. Padmanabhan, Mod. Phys. Lett. A 16, 571 (2001) gr-qc/0007022;

S. Shankaranarayanan, T. Padmanabhan and K. Srinivasan, Class. Quant. Grav. 19, 2671 (2002) gr-qc/0010042.

[5] M.K. Parikh and F. Wilczek, Phys. Rev. Lett. 85, 5042 (2000) hep-th/9907001.

[6] E.C. Vagenas, Phys. Lett. B 503, 399 (2001) hep-th/0012134;

Mod. Phys. Lett. A 17, 609 (2002) hep-th/0108147;

Phys. Lett. B 533, 302 (2002) hep-th/0109108;

Phys. Lett. B 559, 65 (2003) hep-th/0209185.

[7] A.J.M. Medved, Class. Quant. Grav. 19, 589 (2002) hep-th/0110289;

Phys. Rev. D 66, 124009 (2002) hep-th/0207247.

[8] M. Angheben, M. Nadalini, L. Vanzo and S. Zerbini, "Hawking Radiation as Tunneling for Extremal and Rotating Black Holes", arXiv:hep-th/0503081 (2005). 
[9] S.W. Hawking, Phys. Rev. D 14, 2460 (1976).

[10] See, for an example discussion,

J. Preskill, "Do Black Holes Destroy Information", arXiv:hep-th/9209058 (1992).

[11] M.K. Parikh, "Energy Conservation and Hawking Radiation", arXiv:hep-th/0402166 (2004).

[12] M. K. Parikh, Int. J. Mod. Phys. D 13, 2351 (2004) hep-th/0405160.

[13] A.J.M. Medved and E.C. Vagenas, "On Hawking Radiation as Tunneling with BackReaction", arXiv:gr-qc/0504113 (2005).

[14] J.D. Bekenstein, Lett. Nuovo. Cim. 4, 737 (1972);

Phys. Rev. D 7, 2333 (1973);

Phys. Rev. D 9, 3292 (1974).

[15] G.W. Gibbons and S. Hawking, Phys. Rev. D 15, 2738 (1977).

[16] M. Arzano, "Information leak through the quantum horizon", arXiv:hep-th/0504188 (2005).

[17] R.K. Kaul and P. Majumdar, Phys. Lett. 84, 5255 (2000) gr-qc/0002040.

[18] S. Carlip, Class. Quant. Grav. 17, 4175 (2000) gr-qc/0005017.

[19] G. Gour, Phys. Rev. D 66, 104022 gr-qc/0210024.

[20] D.N. Page, "Hawking Radiation and Black Hole Thermodynamics", arXiv:hep-th/0409024 (2004).

[21] M. Domagala and J. Lewandowski, Class. Quant. Grav. 21, 5233 (2004) gr-qc/0407051.

[22] K.A. Meissner, Class. Quant. Grav. 21, 5245 (2004) gr-qc/0407052.

[23] H.A. Kastrup, Phys. Lett. B 413, 267 (1997) gr-qc/9707009.

[24] G. Gour and A.J.M. Medved, Class. Quant. Grav. 20, 3307 (2003) gr-qc/0305018.

[25] A. Chaterjee and P. Majumdar, Phys. Rev. Lett. 92, 141301 (2004) gr-qc/0309026; Pramana 63, 851 (2004) gr-qc/0402061.

[26] A.J.M. Medved, Class. Quant. Grav. 22, 133 (2005) gr-qc/0406044;

"A follow-up to 'Does Nature abhor a logarithm?' (and apparently she dosen't)" arXiv:gr-qc/0411065 (2004).

[27] D.N. Page, Phys. Rev. Lett. 71, 3743 (1993) hep-th/9306083. 